\title{
Book \& New Media Reviews
}

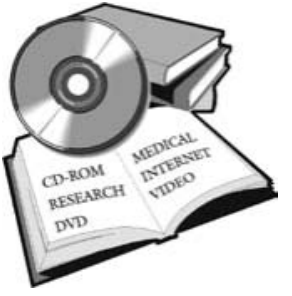

Gastrointestinal and Colorectal Anesthesia

Chandra M. Kumar, Mark Bellamy. Informa Healthcare USA, Inc, New York. 2007, 427 pages. ISBN 0-8493-4073-X

The editors of this newly-published text successfully provide the reader with a comprehensive approach to anesthesia for gastrointestinal and colorectal surgery. Recent developments in the field have been carefully synthesized in this multi-authored book which attempts to cover systematically a topic which often receives rather superficial coverage in more general anesthesia textbooks. Contributing authors include anesthesiologists, intensive care physicians and surgeons. The multidisciplinary structure provides for a relatively comprehensive review of the topic.

The book is divided in 27 chapters which address relevant physiologic considerations, preoperative evaluation, anesthetic management, surgical techniques, and finally, postoperative pain management and perioperative care. Each chapter begins with a brief introduction which reviews the essential physiologic aspects and anatomical considerations, followed by an easy-to-read description of the relevant surgical procedures. The contents span all types of upper and lower abdominal surgery. Related topics are reviewed, including the stress response during surgery, morbidity and outcomes, fast-track surgery for gastrointestinal procedures, the role of critical care units for this surgical patient population, and considerations for organ procurement. Several "hot topics" are reviewed in considerable detail, including insulin-resistance and acute glycemia control, a measure of recovery. The references are comprehensive and up to date.

The text has several limiting elements. Important topics such as organ (especially liver) transplantation and hyperthermic intraperitoneal chemotherapy are conspicuously absent. Further, the chapter devoted to minimally invasive surgery suffers from being overly brief. This is a topic which is attracting growing interest, as every abdominal organ can now be excised through a minimally invasive technique, either through a key-hole or a hand-assisted approach. As a result of novel endoscopic techniques and advances in laparoscopic technology, surgeons and anesthesiologists have been actively modifying their respective practices in order to facilitate the recovery process. Nutritional aspects of perioperative care are discussed in several parts of the book, but the topic is sufficiently complex that a dedicated chapter presenting the most up-to-date concepts would have been beneficial.

Who should read this book? Resident and staff anesthesiologists will consider this a useful reference text within a departmental library. For anesthesiologists and surgeons considering development of clinical pathways for gastrointestinal surgery, and for those embarking on clinical research in this field, this text serves as a good companion resource.

Severine Lauwick MD

Franco Carli MD MPhil

McGill University, Montreal, Canada

\section{Written in Granite: An Illustrated History of the Ether Monument \\ Rafael A. Ortega. Plexus Management, Department of Anesthesiology, Boston University School of Medicine, Boston, 2006. $\$ 100$ or more to www.ether- monument.org. 71 pages. ISBN 0-87270-142-5}

How little did (they) dream that the lapse of a few brief days would herald to the earth the greatest boon ever accorded to the physical welfare of mankind; days of discovery that forever silenced the dreadful shriek of agony which many of us can yet recall in the surgical amphitheatre..., that stilled the moan of the soldier stricken down upon the battlefield, assuaged the pangs of disease, softened the approach of death, and lent a sweet obliviousness in what was once its hour of anguish to all animal existence...

I cannot hope to duplicate this testimonial of Henry J. Bigalow - to the gift mankind was given through the public demonstration of the miracle now known as anesthesia. The testimonial is detailed in this book dedicated to the Ether Monument located in the Boston Public Garden. It is a monument which heralds the discovery of anesthesia to be the end of "surgery as torture". The monument is "rich in history, controversies, and allegories", and its description in 
this book is spectacularly beautiful. I wept when I saw the Ether Monument and read the dedication in this book, as I wept at Vimy and at the Dying Lion monument of Lucerne. Because of this book, I will visit the monument when I am again in Boston.

This book, in my opinion, is a beautiful testament to what we do and to who we are, as anesthesiologists. It is timeless. The pictures are beautifully composed and detailed. The author has not distorted history by his opinion. He has carefully and thoughtfully given credit to those who previously administered analgesics and anesthetics prior to October 16, 1846, including Seishu Hanaoka in 1804.

I am grateful to have been given the opportunity to review this book. Ask for this book, buy it, read it, and share it. It is a wonderful gift for those of us who have been blessed with the knowledge and skills to relieve pain and suffering of humanity.

Gerald V. Goresky MD FRCPC

University of Calgary, Calgary, Canada 